\title{
TOTAL VARIATION AND ERROR ESTIMATES FOR SPECTRAL VISCOSITY APPROXIMATIONS
}

\author{
EITAN TADMOR
}

Dedicated with appreciation to Heinz-Otto Kreiss on his 60th birthday

\begin{abstract}
We study the behavior of spectral viscosity approximations to nonlinear scalar conservation laws. We show how the spectral viscosity method compromises between the total-variation bounded viscosity approximationswhich are restricted to first-order accuracy-and the spectrally accurate, yet unstable, Fourier method. In particular, we prove that the spectral viscosity method is $L^{1}$-stable and hence total-variation bounded. Moreover, the spectral viscosity solutions are shown to be $\mathrm{Lip}^{+}$-stable, in agreement with Oleinik's E-entropy condition. This essentially nonoscillatory behavior of the spectral viscosity method implies convergence to the exact entropy solution, and we provide convergence rate estimates of both global and local types.
\end{abstract}

\section{THE SPECTRAL VISCOSITY APPROXIMATION}

We are concerned here with spectral approximations of the scalar conservation law

$$
\frac{\partial}{\partial t} u(x, t)+\frac{\partial}{\partial x} f(u(x, t))=0, \quad u(x, 0) \equiv u_{0}(x) \in B V .
$$

To single out a unique physically relevant weak solution, (1.1a) is complemented with an entropy condition such that for all convex $U$ 's (e.g., $[7,12])$

$$
\frac{\partial}{\partial t} U(u)+\frac{\partial}{\partial x} F(u) \leq 0, \quad F(u) \equiv \int^{u} U^{\prime}(\xi) f^{\prime}(\xi) d \xi .
$$

We want to solve the $2 \pi$-periodic initial value problem $(1.1 \mathrm{a})-(1.1 \mathrm{~b})$ by spectral methods. To this end, we use an $N$-trigonometric polynomial, $u_{N}(x, t)=$ $\sum_{k=-N}^{N} \hat{u}_{k}(t) e^{i k x}$, to approximate the spectral (or pseudospectral) projection of the exact entropy solution, $P_{N} u$. Starting with $u_{N}(x, 0)=P_{N} u_{0}(x)$, the standard Fourier method reads, e.g., $[5,2,1]$,

$$
\frac{\partial}{\partial t} u_{N}+\frac{\partial}{\partial x} P_{N} f\left(u_{N}\right)=0
$$

Together with one's favorite ODE solver, (1.2) gives a fully discrete spectral method for the approximate solution of (1.1a).

Received by the editor April 9, 1991 and, in revised form, August 16, 1991.

1991 Mathematics Subject Classification. Primary 35L65, 65M06, 65M12, 65M15.

Key words and phrases. Conservation laws, spectral viscosity method, spectral accuracy, total variation, Lipschitz stability, convergence rate estimates.

Research was supported in part by ONR Contract No. N00014-91-J-1076. 
Although the spectral method (1.2) is a spectrally accurate approximation of the conservation law (1.1a), in the sense that its local error does not exceed

$$
\left\|\left(I-P_{N}\right) f\left(u_{N}(\cdot, t)\right)\right\|_{H^{-s}} \leq \text { Const } \cdot N^{-s}\left\|u_{N}\right\|_{L^{2}}, \quad \forall s \geq 0,
$$

the spectral solution, $u_{N}(x, t)$, need not approximate the corresponding entropy solution, $u(x, t)$. Indeed, counterexamples provided in [13,14] show that the spectral approximation (1.2) lacks entropy dissipation, which is inconsistent with the entropy condition (1.1b). Consequently, the spectral approximation (1.2) supports spurious Gibbs oscillations which prevent strong convergence to the exact solution of (1.1).

To suppress these oscillations, without sacrificing the overall spectral accuracy, we consider instead the Spectral Viscosity (SV) approximation

$$
\frac{\partial}{\partial t} u_{N}(x, t)+\frac{\partial}{\partial x} P_{N} f\left(u_{N}(x, t)\right)=\varepsilon_{N} \frac{\partial}{\partial x} Q_{N} * \frac{\partial}{\partial x} u_{N}(x, t) .
$$

The left-hand side of (1.4) is the standard spectral approximation of (1.1a). On the right-hand side, it is augmented by spectral viscosity which consists of the following three ingredients: a vanishing viscosity amplitude of size $\varepsilon_{N} \downarrow 0$, a viscosity-free spectrum of size $m_{N}>>1$, and a viscosity kernel, $Q_{N}(x, t)=$ $\sum_{|k|=m_{N}}^{N} \widehat{Q}_{k}(t) e^{i k x}$, activated only on high wave numbers $|k| \geq m_{N}$, which can be conveniently implemented in the Fourier space as

$$
\varepsilon_{N} \frac{\partial}{\partial x} Q_{N} * \frac{\partial}{\partial x} u_{N}(x, t) \equiv-\varepsilon_{N} \sum_{|k|=m_{N}}^{N} k^{2} \widehat{Q}_{k}(t) \widehat{u}_{k}(t) e^{i k x} .
$$

We deal with real viscosity kernels $Q_{N}(x, t)$ with increasing Fourier coefficients, $\widehat{Q}_{k} \equiv \widehat{Q}_{|k|}$, which satisfy

$$
1-\left(\frac{m_{N}}{|k|}\right)^{2 q} \leq \widehat{Q}_{k}(t) \leq 1, \quad|k| \geq m_{N}, \text { for some fixed } q \geq 1,
$$

and we let the spectral viscosity parameters, $\left(\varepsilon_{N}, m_{N}\right)$, lie in the range

$$
\varepsilon_{N} \sim \frac{1}{N^{\theta} \log N}, \quad m_{N} \sim N^{\frac{\theta}{2 q}}, \quad \theta<1 .
$$

We remark that this choice of spectral viscosity parameters is small enough to retain the formal spectral accuracy of the overall approximation, since

$$
\left\|\varepsilon_{N} \frac{\partial}{\partial x} Q_{N} * \frac{\partial}{\partial x} u_{N}(\cdot, t)\right\|_{H^{-s}} \leq \text { Const } \cdot N^{-\frac{\theta s}{2 q}}\left\|u_{N}(\cdot, t)\right\|_{L^{2}}, \quad \forall s \geq 2 .
$$

At the same time, it is sufficiently large to enforce the correct amount of entropy dissipation that is missing otherwise, when either $\varepsilon_{N}=0$ or $m_{N}=N$. Indeed, it was shown in [13]-[15], [8] that the SV approximation (1.4)-(1.6) $q$ has a bounded entropy production in the sense that

$$
\varepsilon_{N}\left\|\frac{\partial}{\partial x} u_{N}(x, t)\right\|_{L_{\mathrm{loc}}^{2}(x, t)}^{2} \leq \text { Const }
$$

and this together with an $L^{\infty}$-bound imply-by compensated compactness arguments-that the SV approximation $u_{N}$ converges to the unique entropy solution of (1.1). 
Observe that in the limit case $q=\infty$, the SV method (1.4), $(1.5)_{\infty}-(1.6)_{\infty}$, coincides with the usual viscosity approximation, $\frac{\partial}{\partial t} u_{\varepsilon}(x, t)+\frac{\partial}{\partial x} P_{N} f\left(u_{\varepsilon}(x, t)\right)$ $=\varepsilon_{N} \frac{\partial^{2}}{\partial x^{2}} u_{\varepsilon}(x, t)$. But of course, the spectral accuracy (1.7) is lost in this limit case.

In this paper we show that the SV method (1.4)-(1.6) the spectral accuracy (1.7), also shares the essentially nonoscillatory behavior of standard viscosity approximations. In particular, in $\S 3$ we show that the SV solution is total-variation bounded. Moreover, in the genuinely nonlinear case, $f^{\prime \prime}>0$, the SV solution is $\mathrm{Lip}^{+}$-stable, in agreement with Oleinik's E-condition. We conclude that the SV approximation converges to the exact entropy solution of (1.1), and we provide various error estimates.

\section{A TOTAL-VARIATION BOUND}

The presence of spectral viscosity on the right of (1.4) is responsible for a rapid decay of the Fourier coefficients located toward the end of the computed spectrum. This spectral decay result was proved in [8] for the special case of Burgers' equation, $f(u)=\frac{1}{2} u^{2}$, following the argument of [4]. The general case was analyzed by S. Schochet, [11], where it was shown that the following spectral decay estimate holds [11, Theorem 1]:

$$
\begin{aligned}
& \left\|\left(I-P_{k}\right) f\left(u_{N}(\cdot, t)\right)\right\| \\
& \quad \leq K_{s}\left(\frac{k}{N}\right)^{-s} \cdot\left[N^{-s(1-\theta)}+k^{-r} e^{-\varepsilon_{N} \cdot N^{2} t}\right], \quad \forall k \geq N, 0<t \leq T .
\end{aligned}
$$

Here, $r$ and $s$ are related to the smoothness of the data-the initial data $u_{N}(\cdot, 0)$ and the flux $f(\cdot): r \geq 0$ is related to the smoothness of the initial data, $u_{0}$-the initial smoothness being measured by the requirement that

$$
\max _{k<N} k^{r}\left\|\left(I-P_{k}\right) u_{N}(\cdot, 0)\right\| \leq \text { Const; }
$$

and $s$ is any sufficiently large integer, $s \geq s_{0}(r)$, which is related to the degree of smoothness of $f(\cdot)$ measured by the constants $K_{s}$-constants which may depend on $\|f\|_{C^{s}}$ (as well as $\left\|u_{N}\right\|_{L^{\infty}}$ and $\theta$ ), but otherwise are independent of $N$.

The last estimate shows that the discretization error as well as its spatial derivatives, $\partial_{x}^{p}\left(I-P_{N}\right) f\left(u_{N}(\cdot, t)\right)$, become spectrally small independently of whether the underlying entropy solution is smooth or not. Indeed, using the dyadic decomposition $\partial_{x}^{p}\left(I-P_{N}\right) f\left(u_{N}\right) \equiv \sum_{j=0}^{\infty} \partial_{x}^{p} P_{2^{j+1} N}\left(I-P_{2^{j} N}\right) f\left(u_{N}\right)$ and applying the above estimate with $k=2^{j} N, j=0,1, \ldots$, we obtain (consult $[11,(4.9)])$

$$
\begin{aligned}
& \left\|\frac{\partial^{p}}{\partial x^{p}}\left(I-P_{N}\right) f\left(u_{N}(\cdot, t)\right)\right\| \\
& \quad \leq K_{s} \cdot\left[N^{-s(1-\theta)+p}+N^{-r+p} e^{-N^{2-\theta} t}\right], \quad \forall s \geq s_{0} .
\end{aligned}
$$

Remark. As noted in $[11, \S 3]$, the above smoothness requirements are by no means optimal. For the sake of technical convenience, we therefore assume throughout the rest of the paper that the flux, $f(\cdot)$, is sufficiently smooth (e.g., $K_{s}<\infty$ for $s$ large enough so that the first term on the right in $(2.1)_{p}$ is negligible for, say, $s(1-\theta)>2)$. Observe that then the spatial derivatives 
of the truncation error are spectrally small, provided the initial data $u_{N}(\cdot, 0)$ are sufficiently smooth so that the second term on the right of $(2.1)_{p}$ applies with $r>p$ (otherwise, an initial layer of size $<\frac{1}{N}$ may be formed, which is smoothed out exponentially fast once the spectral viscosity becomes effective).

We conclude that the SV approximation is governed by the viscosity-like equation

$$
\frac{\partial}{\partial t} u_{N}(x, t)+\frac{\partial}{\partial x} f\left(u_{N}(x, t)\right)=\varepsilon_{N} \frac{\partial}{\partial x} Q_{N} * \frac{\partial}{\partial x} u_{N}(x, t)+\ldots,
$$

where the missing term ... on the right refers to the spectrally small discretization error $(2.1)_{1}$.

Equation (2.2) is similar to the usual viscosity approximation

$$
\frac{\partial}{\partial t} u_{\varepsilon}(x, t)+\frac{\partial}{\partial x} f\left(u_{\varepsilon}(x, t)\right)=\varepsilon \frac{\partial^{2}}{\partial x^{2}} u_{\varepsilon}(x, t) .
$$

(In fact, the SV method (1.4)-(1.6) $q$ coincides with the viscosity equation (2.3) in the limit case $q=\infty$.) To quantify this similarity, we rewrite (2.2) in the equivalent form

$$
\begin{aligned}
\frac{\partial}{\partial t} u_{N}(x, t)+\frac{\partial}{\partial x} f\left(u_{N}(x, t)\right) & \\
= & \varepsilon_{N} \frac{\partial^{2}}{\partial x^{2}} u_{N}(x, t)-\varepsilon_{N} \frac{\partial}{\partial x} R_{N}(x, t) * \frac{\partial}{\partial x} u_{N}(x, t) \\
& +\frac{\partial}{\partial x}\left(I-P_{N}\right) f\left(u_{N}\right),
\end{aligned}
$$

where

$$
R_{N}(x, t)=\sum_{k=-N}^{N} \widehat{R}_{k}(t) e^{i k x}, \quad \widehat{R}_{k}(t) \equiv \begin{cases}1, & |k|<m_{N}, \\ 1-\widehat{Q}_{k}(t), & |k| \geq m_{N} .\end{cases}
$$

Apart from the spectrally small truncation error on the right, the SV approximation (2.4a) differs from (2.3) by the additional term involving the 'residual kernel', $R_{N}(x, t)$, on its right-hand side. We claim that this kernel is 'sufficiently small'.

Lemma 2.1. Consider the $S V$ kernel $Q_{N}(x, t)$ subject to the $S V$ parameterization $(1.5)_{q}-(1.6)_{q}$. Then $R_{N}(x, t) \equiv D_{N}(x)-Q_{N}(x, t)$ satisfies

$$
\left\|\frac{\partial^{2 s}}{\partial x^{2 s}} R_{N}(\cdot, t)\right\|_{L^{1}} \leq \text { Const } \cdot m_{N}^{2 s} \log N, \quad 0 \leq s \leq q .
$$

Remark. The inequality $(2.5)_{1}$ followed by $(1.6)_{q}$ imply the bound

$$
\varepsilon_{N}\left\|\partial_{x}^{2} R_{N}(\cdot, t)\right\|_{L^{1}} \leq \text { Const }
$$

which plays an essential role in our foregoing discussion. In practice it was found that the latter bound is minimized if we let the monotonically increasing (respectively decreasing) SV coefficients, $\widehat{Q}_{k}$ (respectively $\widehat{R}_{k}$ ), to depend smoothly on the relative wave number $\frac{k}{N}$.

Proof of Lemma 2.1. We first recall

[8, Lemma A.1]: For any symmetric $N$-trigonometric polynomial, $w_{N}(x)=\sum_{k=0}^{N} \hat{w}_{k} \cos k x$, with monotonically decreasing coefficients, $0 \leq \hat{w}_{k} \leq 1$, there holds 


$$
\left\|w_{N}(x)\right\|_{L^{1}} \leq \text { Const } \cdot \log N
$$

(Consideration of the Dirichlet kernel, $D_{N}(x) \equiv 2 \sum_{k=0}^{N} e^{i k x}$, shows that

$$
\text { the last estimate is sharp. ) }
$$

Consider now the symmetric $N$-trigonometric polynomial

$$
\frac{1}{m_{N}^{2 s}} \frac{\partial^{2 s}}{\partial x^{2 s}} R_{N}(x, t)=2 \sum_{k=0}^{N}, \frac{k^{2 s}}{m_{N}^{2 s}} \widehat{R}_{k} \cos k x, \quad s \leq q .
$$

According to the SV parameterization in $(1.5)_{q}$, it has monotonically decreasing Fourier coefficients which satisfy (for $s \leq q$ ) $\frac{k^{2 s}}{m_{N}^{2 s}} \widehat{R}_{k} \equiv \frac{k^{2 s}}{m_{N}^{2 s}}\left[1-\widehat{Q}_{k}\right] \leq 1$. By (2.6), the $L^{1}$-norm of such polynomial does not exceed Const $\cdot \log N$, and $(2.5)_{S}$ follows, for

$$
\begin{aligned}
\left\|\frac{\partial^{2 s}}{\partial x^{2 s}} R_{N}(\cdot, t)\right\|_{L^{1}} & =m_{N}^{2 s}\left\|\sum_{k=-N}^{N} \frac{k^{2 s}}{m_{N}^{2 s}} \widehat{R}_{k}(t) e^{i k x}\right\|_{L^{1}} \\
& \leq \text { Const } \cdot m_{N}^{2 s} \log N, \quad s \leq q .
\end{aligned}
$$

Equipped with Lemma 2.1, one can show now that the SV approximation (2.4)-like the viscosity approximation (2.3) -is $L^{1}$-stable and total-variation bounded. The necessary estimate in this direction is included in the following

Lemma 2.2 ( $L^{1}$-stability). Let $u_{N}$ and $v_{N}$ be two different solutions of the $S V$ approximation (1.4). Then there exist constants $C_{N} \sim N^{-\left(1-\frac{1}{q}\right) \theta}$ such that the following estimate holds:

$$
\begin{aligned}
& \left\|u_{N}(\cdot, t)-v_{N}(\cdot, t)\right\|_{L^{\prime}} \\
& \leq e^{C_{N} t}\left[\left\|u_{N}(\cdot, 0)-v_{N}(\cdot, 0)\right\|_{L^{\prime}}\right. \\
& \left.+\left\|\frac{\partial}{\partial x}\left(I-P_{N}\right)\left[f\left(u_{N}\right)-f\left(v_{N}\right)\right]\right\|_{L^{\prime}(x,[0, t])}\right] .
\end{aligned}
$$

Remark. Taking into account the truncation error spectral decay $(2.1)_{1}$, then inequality (2.7) provides us with the announced $L^{1}$-stability of the form

$$
\begin{aligned}
& \left\|u_{N}(\cdot, t)-v_{N}(\cdot, t)\right\|_{L^{1}} \\
& \quad \leq e^{C_{N} t}\left[\left\|u_{N}(\cdot, 0)-v_{N}(\cdot, 0)\right\|_{L^{1}}+K_{s} \cdot\left(t N^{-s(1-\theta)+1}+N^{-(r+1)+\theta}\right)\right] .
\end{aligned}
$$

Proof of Lemma 2.2. The difference $u_{N}-v_{N}$ satisfies

$$
\begin{aligned}
\frac{\partial}{\partial t}\left(u_{N}\right. & \left.-v_{N}\right)+\frac{\partial}{\partial x}\left(f\left(u_{N}\right)-f\left(v_{N}\right)\right) \\
= & \varepsilon_{N} \frac{\partial^{2}}{\partial x^{2}}\left(u_{N}-v_{N}\right)-\varepsilon_{N} \frac{\partial^{2}}{\partial x^{2}} R_{N} *\left(u_{N}-v_{N}\right) \\
& +\frac{\partial}{\partial x}\left(I-P_{N}\right)\left[f\left(u_{N}\right)-f\left(v_{N}\right)\right] .
\end{aligned}
$$


One may proceed now with an $L^{1}$-estimate in a standard fashion: we integrate against $\operatorname{sgn}\left(u_{N}-v_{N}\right)$, and in view of $(2.5)_{1}$ and $(2.1)_{1}$, we obtain

$$
\begin{aligned}
\frac{d}{d t}\left\|u_{N}(\cdot, t)-v_{N}(\cdot, t)\right\|_{L^{1}} \\
\leq \varepsilon_{N}\left\|\frac{\partial^{2}}{\partial x^{2}} R_{N}(\cdot, t)\right\|_{L^{1}}\left\|u_{N}(\cdot, t)-v_{N}(\cdot, t)\right\|_{L^{1}} \\
\quad+\left\|\frac{\partial}{\partial x}\left(I-P_{N}\right)\left[f\left(u_{N}\right)-f\left(v_{N}\right)\right]\right\|_{L^{1}} \\
\leq C_{N} \cdot\left\|u_{N}(\cdot, t)-v_{N}(\cdot, t)\right\|_{L^{1}}+K_{s} \cdot\left(N^{-s(1-\theta)+1}+N^{-r+1} e^{-N^{2-\theta} t}\right),
\end{aligned}
$$

where according to $(1.6)_{q}$ one has $C_{N} \sim \varepsilon_{N} m_{N}^{2} \log N \sim N^{-\left(1-\frac{1}{q}\right) \theta}$. The assertion (2.7) now follows.

Application of Lemma 2.2 with $v_{N}(\cdot, t)=u_{N}(\cdot+\Delta x, t)$ shows that the total variation of the SV solution,

$$
\left\|u_{N}(\cdot, t)\right\|_{\mathrm{BV}} \equiv \sup _{\Delta x} \frac{1}{\Delta x}\left\|u_{N}(x+\Delta x, t)-u_{N}(x, t)\right\|_{L^{1}},
$$

does not exceed

$$
e^{C_{N} t}\left[\left\|u_{N}(\cdot, 0)\right\|_{\mathrm{BV}}+\left\|\frac{\partial^{2}}{\partial x^{2}}\left(I-P_{N}\right) f\left(u_{N}\right)\right\|_{L^{1}(x,[0, t])}\right] .
$$

In fact, if $q>1$, then $(1.6)_{q}$ implies that $C_{N} \sim N^{-\left(1-\frac{1}{q}\right) \theta} \downarrow 0$, and together with the spectral decay estimate $(2.1)_{2}$ we conclude

Corollary 2.3 (Total-variation boundedness). The SV approximation (1.4)$(1.6)_{q}$ with $q>1$ is essentially nonoscillatory, in the sense that the increase of its initial total variation is $o(1)$,

$$
\begin{aligned}
\left\|u_{N}(\cdot, t)\right\|_{\mathrm{BV}} \leq & \left(1+\mathscr{O}\left(N^{-\left(1-\frac{1}{q}\right) \theta}\right)\right) \\
& \times\left[\left\|u_{N}(\cdot, 0)\right\|_{\mathrm{BV}}+K_{s} \cdot\left(t N^{-s(1-\theta)+2}+N^{-r+\theta}\right)\right] .
\end{aligned}
$$

Remarks. 1. Corollary 2.3 tells us that the SV solution $u_{N}(\cdot, t)$ is total-variation bounded (independently of $N)$, provided its initial data, $u_{N}(\cdot, 0)$, are. $(\mathrm{Ob}$ serve that the BV-smoothness of $u_{N}(\cdot, 0)$ allows us to use $(2.1)_{p}$ with $r \geq \frac{1}{2}$, and hence the contribution of the truncation error, which is bounded from above by the second term on the right of (2.8), is negligible, at least for $\theta<\frac{1}{2}$. For $\frac{1}{2} \leq \theta<1$, however, one might need a slightly stronger assumption regarding the initial smoothness, e.g., $u_{N}(\cdot, 0) \in W^{\frac{3}{2}, 1}$; we shall not explore this issue here since, as noted above, the smoothness requirements for $(2.1)_{p}$ to hold are not optimal to begin with.)

To guarantee the BV-boundedness of $u_{N}(\cdot, 0)$ without sacrificing spectral accuracy, one can preprocess the exact initial data $u_{0}$ prescribed in (1.1a). For example, de la Vallée Poussin's filter,

$$
u_{N}(x, 0)=V P_{N} u_{0} \equiv \sum_{k=-N}^{N} \sigma_{k} \hat{u}_{k}(0) e^{i k x}, \quad \sigma_{k}= \begin{cases}1, & |k| \leq \frac{N}{2}, \\ 2-\frac{2 k}{N}, & |k|>\frac{N}{2},\end{cases}
$$


yields a spectrally accurate approximation of $u_{0}$,

$$
\left\|\left(I-V P_{N}\right) u_{0}\right\|_{H^{-s}} \leq \text { Const } \cdot N^{-\frac{s}{2}}\left\|u_{0}\right\|_{L^{2}}, \quad \forall s \geq 0 ;
$$

moreover, since $\left\|V P_{N} u_{0}\right\|_{\mathrm{BV}} \leq 3\left\|u_{0}\right\|_{\mathrm{BV}}$, it follows from Corollary 2.3 that the

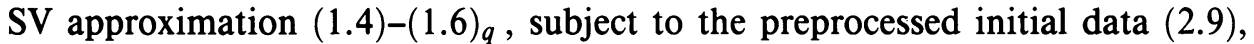
is total-variation bounded (independently of $N$ ) for arbitrary BV-initial data $u_{0}$.

Finally, we note that such initial preprocessing might be necessary, since the unfiltered (pseudo-)spectral projection, $\left\|P_{N} u_{0}\right\|_{\mathrm{BV}}$, may grow as much as $\mathscr{O}(\log N)$ for arbitrary BV-initial data. Of course, it can be avoided if the initial data are smooth enough, say in $H^{1}$, for then $\left\|P_{N} u_{0}\right\|_{\mathrm{BV}} \leq\left\|u_{0}\right\|_{H^{1}}<\infty$.

2. The BV-estimate (2.8) shows how the SV method maintains the delicate tradeoff between spectral accuracy and TV-stability: According to (2.8), the total variation of the SV solution (1.4) $-(1.6)_{q}$ with $q=1$ may grow by a factor of $\mathscr{O}(1)$ times its initial variation; as $q$ increases, this growth factor approaches one-in agreement with the Total-Variation Diminishing (TVD) property of the exact solution, but at the same time, the spectral accuracy estimate (1.7) 'deteriorates'. Thus, the SV method (1.4)-(1.6) $q_{q}$ can be viewed as a compromise between the first-order TVD viscosity approximation (2.3) (which corresponds to $q=\infty)$, and the spectrally accurate, yet unstable, Fourier approximation (1.2) (which corresponds to $q=0$ ).

Similarly, the $L^{1}$-stability (2.7) approaches the $L^{1}$-contraction of the exact entropy solution as we increase the amount of spectral viscosity by letting $q \uparrow \infty$.

3. The total-variation boundedness of the SV solution implies that (a subsequence of ) $u_{N}(x, t)$ converges strongly to a limit $u(x, t)$, which is a weak solution of (1.1a). To conclude that this limit is the unique entropy solution of (1.1), it remains to verify that $u$ satisfies the entropy condition (1.1b). To this end, we multiply $(2.4 \mathrm{a})$ by $U^{\prime}\left(u_{N}\right)$, obtaining

$$
\begin{aligned}
\frac{\partial}{\partial t} U\left(u_{N}\right)+\frac{\partial}{\partial x} F\left(u_{N}\right)= & \frac{\partial}{\partial x}\left(\varepsilon_{N} U^{\prime}\left(u_{N}\right) \frac{\partial u_{N}}{\partial x}\right)-\varepsilon_{N} U^{\prime \prime}\left(u_{N}\right)\left(\frac{\partial u_{N}}{\partial x}\right)^{2} \\
& +\frac{\partial}{\partial x}\left(\varepsilon_{N} u_{N} R_{N} * \frac{\partial u_{N}}{\partial x}\right)-\varepsilon_{N} \frac{\partial u_{N}}{\partial x} R_{N} * \frac{\partial u_{N}}{\partial x} \\
= & \frac{\partial}{\partial x} \mathrm{I}+\mathrm{II}+\frac{\partial}{\partial x} \mathrm{III}+\mathrm{IV} .
\end{aligned}
$$

Since the entropy function $U$ is convex, II $\leq 0$. This, together with the straightforward estimates (based on $(2.5)_{0},(2.8)$ and $(3.3)_{0}$ below)

$$
\begin{aligned}
&\|\mathrm{I}\|_{L^{1}}+\|\mathrm{III}\|_{L^{1}} \leq \varepsilon_{N}\left\|R_{N}\right\|_{L^{1}}\left\|u_{N}\right\|_{L^{\infty}}\left\|u_{N}\right\|_{\mathrm{BV}} \leq \text { Const } \cdot \varepsilon_{N} \log N\left\|u_{0}\right\|_{\mathrm{BV}} \rightarrow 0, \\
&\|\mathrm{IV}\|_{L^{1}} \leq \varepsilon_{N}\left\|R_{N}\right\|_{L^{\infty}}\left\|u_{N}\right\|_{\mathrm{BV}}^{2} \leq \text { Const } \cdot \varepsilon_{N} m_{N} \log N\left\|u_{0}\right\|_{\mathrm{BV}}^{2} \quad \rightarrow 0,
\end{aligned}
$$

imply that $u=\lim u_{N}$ satisfies the entropy inequality (1.1b), and convergence of (the whole sequence of ) $u_{N}$ to the entropy solution follows.

4. The total-variation bound indicated above implies the usual decay

$$
\left|\hat{u}_{k}(t)\right| \leq \frac{\left\|u_{N}(\cdot, t)\right\|_{\mathrm{BV}}}{|k|+1},
$$


which in turn implies, by the Parseval identity, an $L^{2}(x)$-bounded entropy production estimate (uniformly in time) of the type indicated earlier in (1.8),

$$
\frac{1}{N}\left\|\frac{\partial}{\partial x} u_{N}(\cdot, t)\right\|_{L_{\mathrm{loc}}^{2}(x)}^{2} \leq \text { Const }, \quad 0 \leq t \leq T \text {. }
$$

\section{Convergence RAte estimates}

In this section we restrict our attention to the genuinely nonlinear conservation law (1.1) where $f^{\prime \prime} \geq \alpha>0$.

We say that a family of approximate solutions $\left\{u_{N}(x, t)\right\}$ is $\mathrm{Lip}^{+}$-stable, if there exists a constant (independent of $N$ ), such that the following estimate is fulfilled ${ }^{1}$ :

$$
\left\|u_{N}(\cdot, t)\right\|_{\text {Lip }^{+}} \leq \text {Const }_{T}, \quad 0 \leq t \leq T .
$$

Recall that the viscosity approximation $u_{\varepsilon}$ as well as the entropy solution of the nonlinear conservation law (1.1) with $f^{\prime \prime} \geq \alpha>0$ satisfy Oleinik's E-condition, e.g., $[7,15]$,

$$
\left\|u_{\varepsilon}(\cdot, t)\right\|_{\text {Lip }^{+}} \leq \frac{1}{\left\|u_{0}\right\|_{\text {Lip }^{+}}^{-1}+\alpha t}, \quad t \geq 0 .
$$

In particular, they are $\mathrm{Lip}^{+}$-stable as long as their initial data $u_{0}$ are $\mathrm{Lip}^{+}$bounded. We want to show that the SV approximation (1.4) is also Lip $^{+}$-stable.

We remark that the BV-bound (2.8) does not exclude the possibility of small high-frequency oscillations. (By conservation, Lip $^{+}$- implies BV-stability, but not vice versa). Such 'unphysical' oscillations may violate the Lip ${ }^{+}$-stability of the SV solution. In order to prevent such $\mathrm{Lip}^{+}$-unstable oscillations, we therefore need to slightly increase the amount of spectral viscosity. We achieve this (without sacrificing formal spectral accuracy) by requiring the spectral viscosity parameters to lie in the range $(1.5)_{q}-(1.6)_{q}$ with $q \geq \frac{3}{2}$.

As before, the $\mathrm{Lip}^{+}$-stability of the SV method hinges on the (small) size of the 'residual' kernel, $R_{N}(x, t)$, which distinguishes the $\mathrm{SV}$ approximation (2.4a) from the $\mathrm{Lip}^{+}$-stable viscosity approximation (2.3). To this end, we first state

Lemma 3.1. Consider the $S V$ kernel $Q_{N}(x, t)$ subject to the $S V$ parameterization $(1.5)_{q}-(1.6)_{q}$. Then $R_{N}(x, t) \equiv D_{N}(x)-Q_{N}(x, t)$ satisfies

$$
\left\|\frac{\partial^{2 s}}{\partial x^{2 s}} R_{N}(\cdot, t)\right\|_{L^{\infty}} \leq \text { Const } \cdot m_{N}^{2 s+1} \log N, \quad 0 \leq s \leq q-\frac{1}{2} \text {. }
$$

Proof. By $(1.5)_{q}, \widehat{R}_{k} \equiv 1-\widehat{Q}_{k} \leq\left(\frac{m_{N}}{|k|}\right)^{2 q}$. The lemma follows from the straightforward estimate

$$
\begin{aligned}
\left\|\frac{\partial^{2 s}}{\partial x^{2 s}} R_{N}(\cdot, t)\right\|_{L^{\infty}} & \leq \sum_{|k| \leq N}|k|^{2 s}\left|\widehat{R}_{k}\right| \leq \sum_{|k|<m_{N}}|k|^{2 s}+\sum_{|k| \geq m_{N}}|k|^{2 s}\left(\frac{m_{N}}{|k|}\right)^{2 q} \\
& \leq \text { Const } \cdot m_{N}^{2 s+1}+\text { Const } \cdot m_{N}^{2 q} \cdot\left\{\begin{array}{ll}
m_{N}^{2 s-2 q+1} & \text { if } s<q-\frac{1}{2} \\
\log N & \text { if } s=q-\frac{1}{2}
\end{array}\right\} \\
& \leq \text { Const } \cdot m_{N}^{2 s+1} \log N . \quad \square
\end{aligned}
$$

\footnotetext{
${ }^{1}$ We let $\|\phi\|_{\text {Lip }},\|\phi\|_{\text {Lip }^{+}}$and $\|\phi\|_{\text {Lip' }^{\prime}}$ denote respectively, ess sup $x \neq y=(\phi(x)-\phi(y)) /(x-y) \mid$, ess $\sup _{x \neq y}[(\phi(x)-\phi(y)) /(x-y)]_{+}$and $\sup _{\psi}\left(\phi-\hat{\phi}_{0}, \psi\right) /\|\psi\|_{\text {Lip }}$.
} 
Equipped with Lemma 3.1, we now turn to the $\mathrm{Lip}^{+}$-stability proof of the SV method, stating

Lemma 3.2 ( Lip $^{+}$-stability). The $S V$ approximation with $q>\frac{3}{2}$ satisfies the Lip $^{+}$-stability estimate

$$
\left\|u_{N}(\cdot, t)\right\|_{\mathrm{Lip}^{+}} \leq \frac{1+\sqrt{\frac{c_{N}}{\alpha}}}{\left\|u_{N}(\cdot, 0)\right\|_{\mathrm{Lip}^{+}}^{-1}+\alpha_{N} t}, \quad \alpha_{N} \equiv \alpha \frac{\tanh \left(\sqrt{\alpha c_{N}} t\right)}{\sqrt{\alpha c_{N}} t} \sim \alpha,
$$

where the vanishingly small constants, $c_{N}$, are given by

$$
c_{N} \sim N^{-\theta\left(1-\frac{3}{2 q}\right)} \cdot\left\|u_{N}(\cdot, 0)\right\|_{\mathrm{BV}}+K_{s} \cdot\left(N^{-s(1-\theta)+\frac{5}{2}}+N^{-r+\frac{1}{2}+\theta}\right) .
$$

Remark. The constants $c_{N}$ involved in the Lip ${ }^{+}$-bound (3.4) have two major contributions: the second term on the right represents an upper bound of the truncation error, which is spectrally small-provided the initial data $u_{N}(\cdot, 0)$ are sufficiently smooth, say $u_{N}(\cdot, 0) \in W^{2,1}$ so that (3.4) holds with $r \geq \frac{3}{2}$ (otherwise, an initial layer may be formed, after which the spectral viscosity becomes effective and drives the truncation error spectrally small). Apart from this spectrally small contribution, we have $c_{N} \sim N^{-\theta\left(1-\frac{3}{2 q}\right)}$; here we observe that, as before, when the amount of spectral viscosity increases with $q$, the $\mathrm{Lip}^{+}$-bound in (3.4) becomes tighter, in agreement with (3.2), and in particular, the two $\mathrm{Lip}^{+}$-bounds coincide in the fully viscous limit $q=\infty$.

Proof of Lemma 3.2. Differentiation of (2.4a) yields, for $w_{N}(x, t) \equiv \frac{\partial}{\partial x} u_{N}(x, t)$,

$$
\begin{aligned}
\frac{\partial}{\partial t} w_{N}(x, t) & +f^{\prime}\left(u_{N}(x, t)\right) \frac{\partial}{\partial x} w_{N}(x, t)+f^{\prime \prime}\left(u_{N}(x, t)\right) w_{N}^{2}(x, t) \\
= & \varepsilon_{N} \frac{\partial^{2}}{\partial x^{2}} w_{N}(x, t)-\varepsilon_{N} \frac{\partial^{2}}{\partial x^{2}} R_{N} * w_{N}(x, t)+\frac{\partial^{2}}{\partial x^{2}}\left(I-P_{N}\right) f\left(u_{N}\right),
\end{aligned}
$$

which implies that $\left\|u_{N}(\cdot, t)\right\|_{\mathrm{Lip}^{+}}=\max _{x}\left[w_{N}(x, t)_{+}\right]$satisfies the differential inequality

$$
\begin{aligned}
& \frac{d}{d t}\left\|u_{N}(\cdot, t)\right\|_{\mathrm{Lip}^{+}}+\alpha\left\|u_{N}(\cdot, t)\right\|_{\mathrm{Lip}^{+}}^{2} \\
& \quad \leq \varepsilon_{N}\left\|\frac{\partial^{2}}{\partial x^{2}} R_{N}(\cdot, t)\right\|_{L^{\infty}} \cdot\left\|u_{N}(\cdot, t)\right\|_{\mathrm{BV}}+\left\|\frac{\partial^{2}}{\partial x^{2}}\left(I-P_{N}\right) f\left(u_{N}\right)\right\|_{L^{\infty}} \\
& \quad \equiv \mathrm{I}_{N}+\mathrm{II}_{N} .
\end{aligned}
$$

We recall that according to $(3.3)_{1}$,

$$
\begin{aligned}
I_{N} & =\varepsilon_{N}\left\|\frac{\partial^{2}}{\partial x^{2}} R_{N}(\cdot, t)\right\|_{L^{\infty}}\left\|u_{N}\right\|_{L^{\infty}(\mathrm{BV},[0, t])} \\
& \leq \text { Const } \cdot N^{-\theta\left(1-\frac{3}{2 q}\right)} \cdot\left\|u_{N}\right\|_{L^{\infty}(\mathrm{BV},[0, t])}
\end{aligned}
$$

moreover, according to $(2.1)_{2}$, together with Sobolev's inequality, we have

$$
\mathrm{II}_{N}=\left\|\frac{\partial^{2}}{\partial x^{2}}\left(I-P_{N}\right) f\left(u_{N}\right)\right\|_{L^{\infty}} \leq K_{s} \cdot\left(N^{-s(1-\theta)+\frac{5}{2}}+N^{-r+\frac{5}{2}} e^{-N^{2-\theta} t}\right) .
$$

Equipped with (3.6a)-(3.6b) we return to (3.5), obtaining

$$
\frac{d}{d t}\left\|u_{N}(\cdot, t)\right\|_{\mathrm{Lip}^{+}}+\alpha\left\|u_{N}(\cdot, t)\right\|_{\mathrm{Lip}^{+}}^{2} \leq c_{N}, \quad c_{N} \equiv \mathrm{I}_{N}+\mathrm{II}_{N} \downarrow 0,
$$


which in turn implies the desired Lip $^{+}$-stability of $u_{N}$,

$$
\begin{aligned}
\left\|u_{N}(\cdot, t)\right\|_{\text {Lip }^{+}} & \leq \frac{c_{N} \tanh \left(\sqrt{\alpha c_{N}} t\right)+\sqrt{\alpha c_{N}}\left\|u_{N}(\cdot, 0)\right\|_{\text {Lip }^{+}}}{\sqrt{\alpha c_{N}}+\alpha \tanh \left(\sqrt{\alpha c_{N}} t\right)\left\|u_{N}(\cdot, 0)\right\|_{\text {Lip }^{+}}} \\
& \leq \frac{1+\sqrt{\frac{c_{N}}{\alpha}}}{\left\|u_{N}(\cdot, 0)\right\|_{\text {Lip }^{+}}^{-1}+\alpha_{N} t}, \quad \alpha_{N} \equiv \alpha \frac{\tanh \left(\sqrt{\alpha c_{N}} t\right)}{\sqrt{\alpha c_{N}} t} \sim \alpha .
\end{aligned}
$$

We now recall the main result of [9] (see also [16]), which deals with the convergence rate of $\mathrm{Lip}^{+}$-stable approximations.

Theorem 3.3 [9]. Let $\left\{u_{N}(x, t)\right\}$ be a family of Lip $^{+}$-stable approximate solutions of the conservation law (1.1), with $\mathrm{Lip}^{+}$-bounded initial data. Assume that $\left\{u_{N}(x, t)\right\}$ are Lip'-consistent of order $\varepsilon$,

$$
\begin{aligned}
\left\|u_{N}(x, 0)-u_{0}(x)\right\|_{\text {Lip }^{\prime}} & +\left\|\frac{\partial}{\partial t} u_{N}(\cdot, t)+\frac{\partial}{\partial x} f\left(u_{N}(\cdot, t)\right)\right\|_{\text {Lip }^{\prime}} \\
& \leq \text { Const }_{T} \cdot \varepsilon, \quad 0 \leq t \leq T .
\end{aligned}
$$

Then the following error estimates hold:

(3.8a) $\left\|u_{N}(\cdot, t)-u(\cdot, t)\right\|_{W-s, p} \leq$ Const $\cdot \varepsilon^{\frac{s p+1}{2 p}}, \quad 1 \leq p<\infty,-\frac{1}{p} \leq s \leq 1$;

(3.8b) $\left|u_{N}(x, t)-u(x, t)\right| \leq C_{1} \cdot \varepsilon^{\frac{1}{3}}, \quad 0 \leq t \leq T$;

(3.8c) $\left|u_{N}(x, t) * \psi_{r}-u(x, t)\right| \leq C_{r} \cdot \varepsilon^{\frac{r}{r+2}}, \quad 1 \leq r<\infty$.

Here, $\psi_{r}$ is any rth-order mollifier, and the constants $C_{r} \sim 1+\|u(\cdot, t)\|_{W_{\mathrm{loc}}^{r . \infty}}^{\frac{2}{r+2}}$ measure the local smoothness of the entropy solution in the $\mathscr{O}\left(\varepsilon^{\frac{1}{r+2}}\right)$-neighborhood of $x$.

We have shown that the SV approximation is $\mathrm{Lip}^{+}$-stable and hence convergent to the exact entropy solution of (1.1). To estimate the convergence rate with the help of Theorem 3.3, it remains to verify the order of its Lip'-consistency. To this end, we note

1. The initial data, $u_{N}(x, 0)=P_{N} u_{0}$, are Lip'-consistent of order $\sim \frac{1}{N}$ with the BV-initial data $u_{0}$, for by $(2.10)$,

$$
\left\|P_{N} u_{0}-u_{0}\right\|_{\text {Lip }^{\prime}} \leq \sum_{|k|>N}\left|\frac{\hat{u}_{k}(0)}{k}\right| \leq \frac{1}{N}\left\|u_{0}\right\|_{B V} .
$$

2. The SV approximation (1.4) is $\mathrm{Lip}^{\prime}$-consistent with the conservation law (1.1a) of order $\varepsilon_{N} \log N \sim N^{-\theta}$, for

$$
\begin{aligned}
\left\|\frac{\partial}{\partial t} u_{N}(\cdot, t)+\frac{\partial}{\partial x} f\left(u_{N}(\cdot, t)\right)\right\|_{\mathrm{Lip}^{\prime}} & \leq\left\|\varepsilon_{N} \frac{\partial}{\partial x} Q_{N} * \frac{\partial}{\partial x} u_{N}(\cdot, t)\right\|_{\mathrm{Lip}^{\prime}} \\
\leq \varepsilon_{N}\left\|Q_{N}(\cdot, t)\right\|_{L^{1}}\left\|u_{N}(\cdot, t)\right\|_{\mathrm{BV}} & \leq \text { Const } \cdot N^{-\theta}\left\|u_{N}(\cdot, 0)\right\|_{\mathrm{BV}}
\end{aligned}
$$

Here, the first inequality follows from (2.2) by ignoring the spectrally small discretization error (2.1); the second is an obvious use of Young's inequality; and the third inequality uses $(2.5)_{0}$ and (2.6), which show that the $L^{1}$-norm of the viscosity kernel, $Q_{N}(\cdot, t) \equiv D_{N}(\cdot)-R_{N}(\cdot, t)$, does not exceed Const $\cdot \log N$. 
In summary, we find that the $\mathrm{Lip}^{+}$-stable SV approximation (1.4) is $\mathrm{Lip}^{\prime}$ consistent of order $\varepsilon \sim N^{-\theta}$, and using Theorem 3.3, we conclude

Theorem 3.4 (Convergence rate estimates). Consider the $2 \pi$-periodic nonlinear conservation law (1.1) with $\mathrm{Lip}^{+}$initial data. Then the SV approximation (1.4), (1.5) $)_{q}-(1.6)_{q}$ with $q \geq \frac{3}{2}$, converges to the entropy solution of (1.1), and the following error estimates hold for $1 \leq p, r<\infty,-1 / p \leq s \leq 1$ :

$$
\begin{aligned}
& \left\|u_{N}(\cdot, t)-u(\cdot, t)\right\|_{W-s, p} \leq \text { Const } \cdot N^{-\frac{s p+1}{2 p} \theta}, \quad 0<t_{0} \leq t \leq T \\
& \left|u_{N}(x, t)-u(x, t)\right| \leq C_{1} \cdot N^{-\frac{\theta}{3}}, \quad 0<t_{0} \leq t \leq T \\
& \left|u_{N}(x, t) * \psi_{r}-u_{N}(x, t)\right| \leq C_{r} \cdot N^{-\frac{r}{r+2} \theta}, \quad 0<t_{0} \leq t \leq T
\end{aligned}
$$

Remarks. 1. Theorem 3.4 requires the initial data of the SV method, $u_{N}(x, 0)$, to be $\mathrm{Lip}^{+}$-bounded independently of $N$. Consequently, one might need to preprocess the prescribed initial data $u_{0}$ unless they are smooth enough to begin with. The de la Vallee Poussin preprocessing in (2.9) will guarantee this requirement for arbitrary $\mathrm{Lip}^{+}$-bounded initial data $u_{0}$.

2. The error estimates (3.10), (3.11) are not uniform in time as $t_{0} \downarrow 0$, unless the initial data are sufficiently smooth to guarantee the uniformity (in time) of the Lip $^{+}$bound (3.4); consult the remark following Lemma 3.2. For arbitrary $\mathrm{Lip}^{+}$initial data, $u_{0}$, an initial layer may be formed, after which the spectral viscosity becomes effective and guarantees the spectral decay of the discretization error indicated in $(2.1)_{p}$.

3. According to (3.11) and (3.12), the pointwise convergence rate of the SV solution in smooth regions of the entropy solution is of order $\sim N^{-\frac{1}{3}}$, and by postprocessing the SV solution, this convergence rate can be made arbitrarily close to $N^{-1}$. In fact, numerical experiments reported in [14] show that by postprocessing the SV solution using the spectrally accurate mollifier of [3], $\psi_{r}(x)=\psi_{0}(x) D_{n}(x), n \sim\left[\varepsilon_{N}^{-\frac{1}{r+2}}\right]$, we recover the pointwise values in smooth regions of the entropy solution within spectral accuracy.

4. According to $(3.10)$ with $(s, p)=(0,1)$, the SV approximation has an $L^{1}$-convergence rate of order $\sim N^{-\frac{1}{2}}$, in agreement with [11, §5]. This corresponds to the usual $L^{1}$-convergence rate of order $\frac{1}{2}$ for monotone difference approximations, $[6,10]$.

\section{BIBLIOGRAPHY}

1. C. Canuto, M. Y. Hussaini, A. Quarteroni, and T. Zang, Spectral methods in fluid dynamics, Springer-Verlag, New York, 1988.

2. D. Gottlieb and S. Orszag, Numerical analysis of spectral methods: Theory and applications, CBMS-NSF Regional Conference Series in Applied Mathematics 25, SIAM, Philadelphia, PA, 1977.

3. D. Gottlieb and E. Tadmor, Recovering pointwise values of discontinuous data within spectral accuracy, in "Progress in Supercomputing in Computational Fluid Dynamics," Progress in Scientific Computing, Vol. 6 (E. M. Murman and S. S. Abarbanel, eds.), Birkhäuser, Boston, 1985, pp. 357-375.

4. H.-O. Kreiss, Fourier expansions of the solutions of Navier-Stokes equations and their exponential decay rate, Analyse Mathématique et Appl., Gauthier-Villars, Paris, 1988, pp. 245-262.

5. H.-O. Kreiss and J. Oliger, Comparison of accurate methods for the integration of hyperbolic equations, Tellus 24 (1972), 199-215. 
6. N. N. Kuznetsov, On stable methods for solving non-linear first order partial differential equations in the class of discontinuous functions, Topics in Numerical Analysis III (Proc. Roy. Irish Acad. Conf., J. J. H. Miller, ed.), Academic Press, London, 1977, pp. 183-197.

7. P. D. Lax, Hyperbolic systems of conservation laws and the mathematical theory of shock waves, Regional Conf. Series in Appl. Math., SIAM, Philadelphia, PA, 1973.

8. Y. Maday and E. Tadmor, Analysis of the spectral viscosity method for periodic conservation laws, SIAM J. Numer. Anal. 26 (1989), 854-870.

9. H. Nessyahu and E. Tadmor, The convergence rate of approximate solutions for nonlinear conservation laws, SIAM J. Numer. Anal. 29 (1992), in press.

10. R. Sanders, On convergence of monotone finite difference schemes with variable spatial differencing, Math. Comp. 40 (1983), 91-106.

11. S. Schochet, The rate of convergence of spectral-viscosity methods for periodic scalar conservation laws, SIAM J. Numer. Anal. 27 (1990), 1142-1159.

12. J. Smoller, Shock waves and reaction-diffusion equations, Springer-Verlag, New York, 1983.

13. E. Tadmor, Convergence of spectral methods for nonlinear conservation laws, SIAM J. Numer. Anal. 26 (1989), 30-44.

14. __ Shock capturing by the spectral viscosity method, Comput. Methods Appl. Mech. Engrg. 78 (1990), 197-208.

15. S_ Semi-discrete approximations to nonlinear systems conservation laws; consistency and $L^{\infty}$-stability imply convergence, ICASE Report No. 88-41.

16. __ Local error estimates for discontinuous solutions of nonlinear hyperbolic equations, SIAM J. Numer. Anal. 28 (1991), 891-906.

School of Mathematical Sciences, Tel-Aviv University, Tel-Aviv 69978, IsRael E-mail address: tadmor@taurus.bitnet 